\title{
Rhubarb extract prevents hepatic inflammation induced by acute alcohol intake, an effect related to the modulation of the gut microbiota
}

\author{
Audrey M. Neyrinck ${ }^{1 *}$, Usune Etxeberria ${ }^{1,2 *}$, Bernard Taminiau ${ }^{3}$, Georges Daube $^{3}$, \\ Matthias Van Hul ${ }^{1,4}$, Amandine Everard ${ }^{1,4}$, Patrice D. Cani ${ }^{1,4}$, Laure B. Bindels ${ }^{1}$ \\ and Nathalie M. Delzenne ${ }^{1}$ \\ ${ }^{1}$ Louvain Drug Research Institute, Metabolism and Nutrition Research Group, Université catholique de Louvain, \\ Brussels, Belgium \\ ${ }^{2}$ Department of Nutrition, Food Science and Physiology, University of Navarra, Pamplona, Spain \\ ${ }^{3}$ Fundamental and Applied Research for Animal and Health-Department of Food Sciences, Université de Liège, \\ Liège, Belgium \\ ${ }^{4}$ Walloon Excellence in Life sciences and BIOtechnology (WELBIO), Louvain Drug Research Institute, Brussels, \\ Belgium
}

\begin{abstract}
Scope: Binge consumption of alcohol is an alarming global health problem. Acute ethanol intoxication is characterized by hepatic inflammation and oxidative stress, which could be promoted by gut barrier function alterations. In this study, we have tested the hypothesis of the hepatoprotective effect of rhubarb extract in a mouse model of binge drinking and we explored the contribution of the gut microbiota in the related metabolic effects.

Methods and results: Mice were fed a control diet supplemented with or without $0.3 \%$ rhubarb extract for 17 days and were necropsied $6 \mathrm{~h}$ after an alcohol challenge. Supplementation with rhubarb extract changed the microbial ecosystem (assessed by 16S rDNA pyrosequencing) in favor of Akkermansia muciniphila and Parabacteroides goldsteinii. Furthermore, it improved alcohol-induced hepatic injury, downregulated key markers of both inflammatory and oxidative stresses in the liver tissue, without affecting significantly steatosis. In the gut, rhubarb supplementation increased crypt depth, tissue weight, and the expression of antimicrobial peptides. Conclusions: These findings suggest that some bacterial genders involved in gut barrier function, are promoted by phytochemicals present in rhubarb extract, and could therefore be involved in the modulation of the susceptibility to hepatic diseases linked to acute alcohol consumption.
\end{abstract}

\section{Keywords:}

Akkermansia muciniphila / Alcoholic liver disease / Antimicrobial peptides / Gut barrier / Microbiota / Parabacteroides goldsteinii / Steatosis

Additional supporting information may be found in the online version of this article at the publisher's web-site

\author{
Received: December 15, 2015 \\ Revised: February 26, 2016 \\ Accepted: March 14, 2016
}

Correspondence: Nathalie M. Delzenne

E-mail: nathalie.delzenne@uclouvain.be

\footnotetext{
Abbreviations: ALAT, alanine aminotransferase; ALD, alcoholic liver disease; LPS, lipopolysaccharides; PIA2g2, phospholipase A2 group-II; qPCR, quantitative PCR; Reglll $\gamma$, regenerating isletderived 3-gamma; ROS, reactive oxygen species; TBARS, thiobarbituric acid reactive substances; TLR, toll-like receptor; TNF- $\alpha$, tumor necrosis factor $\alpha$
}

\section{Introduction}

Alcohol abuse represents a risk factor for numerous diseases. In particular, "binge drinking" is on the rise at an alarming rate worldwide. A binge is defined by the National Institute on Alcohol Abuse and Alcoholism as consumption of five and four drinks for men and women, respectively, in $2 \mathrm{~h}$

\footnotetext{
*These authors contributed equally to this work.

Colour online: See article online to view Figs. 1-6 in colour.
} 
producing a blood ethanol level above $0.08 \%$ (80 mg/dL) [1]. The ultimate consequence of heavy acute and/or chronic long-term alcohol consumption is alcoholic liver disease (ALD) that generates an important financial burden for the health care system due to the associated morbidity [2]. Pathogenesis of fatty liver by alcohol consumption is known to be complex and to have a multifactorial origin [3]. It is widely described that alcohol metabolism per se causes fat accumulation in the liver [4]. A "two-hit" hypothesis has been proposed as the major contributor for alcohol-induced steatosis [5]. The first one would involve the oxidation of ethanol to produce acetaldehyde by cytosolic alcohol dehydrogenases, followed by further oxidation toward acetate. Both processes are coupled to the reduction of $\mathrm{NAD}^{+}$to $\mathrm{NADH}$ and ultimately contribute to steatosis [6]. However, while chronic alcohol-induced liver injury has been a focus of interest for many years, the mechanisms of acute ethanol intake and metabolic consequences are still poorly understood. A recent study revealed that the alcohol-mediated acetylation of the transcription of carbohydrate responsive element binding protein (ChREBP) plays an important role in steatosis under binge drinking condition [7].

Furthermore, it is well known that acute alcohol intoxication leads to mucosal damage, quantitative and qualitative alterations of the gut microbiota composition (i.e. dysbiosis), and increased gut permeability, resulting in the translocation of endotoxins (namely, lipopolysaccharides (LPS)) and other bacterial products into the portal blood flow $[1,8]$. This altered gut barrier function will promote the delivery of toll-like receptor (TLR) ligands like LPS to the hepatic macrophages known as the Kupffer cells. In the liver, bacterial products stimulate the release of pro-inflammatory mediators such as reactive oxygen species (ROS), leukotrienes, chemokines, and cytokines (e.g. tumor necrosis factor $\alpha$ (TNF- $\alpha$ ) and IL$1 \beta)$ exerting deleterious effects on hepatocytes and resulting in inflammatory infiltration and fibrosis in the liver [8]. Thus, acute alcohol exposure can cause a "perfect storm" that favors inflammatory liver damage. In accordance with these observations, data suggest that blocking signaling and pro-oxidant producing enzymes in rodents via genetic alterations also protect against steatosis, without altering ethanol metabolism [9]. Among the other factors, data suggests that the gut microbiota is a potential important target and an actor involved in the gut barrier alteration upon alcohol drinking $[10,11]$. In humans, our recent results suggest that the gut microbiota is an actor in the gut barrier and in behavioral disorders induced upon alcohol dependence [12].

From a therapeutic point of view, nutritional strategies targeting the experimental ALD, such as anti-inflammatory and antioxidant nutrients, are of growing interest as promising approaches showing fewer side effects than synthetic drugs [13]. There is compelling evidence supporting the beneficial effects of plant-derived compounds on the prevention and treatment of numerous illnesses, including ALD [14-16]. For instance, the rhubarb extract (from Rheum palmatum L./ Rheum officinale) has been classified as a natural herb rich in anthraquinone derivatives with hepatoprotective and anticancer effects $[17,18]$. Intriguingly, the potential hepatoprotective effects of rhubarb extract on acute alcohol-induced lesions have not been investigated so far. Therefore, the present study was designed to use the potential benefits of rhubarb extract administration on liver damages in a mouse model of binge drinking, in order to evaluate the link between gut microbiota, gut barrier, and hepatic metabolic disorders and inflammation.

\section{Material and methods}

\subsection{Animals and diet intervention}

Male C57BL/6J mice (12-wk-old) were purchased from Charles River Laboratories (France) and maintained in specific pathogen-free environment. Animals were housed in groups of three or four mice per cage in a controlled environment (12-h daylight cycle) with free access to food and water. Animal experiments were approved and performed in accordance with the guidelines of the local ethics committee. Housing conditions were as specified by the Belgian Law of May 29, 2013, on the protection of laboratory animals (Agreement LA 1230314). The agreement of the animal experiments performed in this study was given by the local Ethical Committee under the specific number 2010/UCL/MD/022. Two independent experiments were performed following the same protocol.

After 1 wk of acclimatization, mice were randomly assigned into two groups: a group fed with a standard diet (AIN93M, Research Diet, New Brunswick, NJ) and a group fed the standard diet supplemented with $0.3 \%$ of a rhubarb extract for 17 days. Rhubarb extract was supplied by ORTIS Laboratoires (Elsenborn, Belgium). The composition of this extract was given in Supporting Information Table 1 with the most important anthraquinone derivatives; the percentage of total anthracenic derivatives (expressed as rhein) was $6.48 \%$ (expressed as wet weight) and 6.87\% (expressed as dry weight). The diets were composed of $14 \mathrm{~g} / 100 \mathrm{~g}$ protein (obtained from casein); $77 \mathrm{~g} / 100 \mathrm{~g}$ carbohydrates (obtained from corn starch, maltodextrin 10, sucrose, and cellulose BW200) and $4 \mathrm{~g} / 100 \mathrm{~g}$ of lipids (obtained from soybean oil). The caloric content of the diet was $3.85 \mathrm{kcal} / \mathrm{g}$.

\subsubsection{Alcohol challenge}

After 17 days of dietary treatments, mice were fasted overnight and minimum 14 mice per group received an ethanol solution $(30 \% \mathrm{w} / \mathrm{v}, 6 \mathrm{~g} / \mathrm{kg}$ body weight), the remaining mice (minimum 8 mice per group) received water by intragastric gavage (sham treatment). Mice were anaesthetized with isoflurane gas (Abbot, Ottignies, Belgium) $6 \mathrm{~h}$ after the gavage with alcohol or water. Blood samples were harvested for further analysis. Mice were necropsied after 
cervical dislocation. Cecal content and tissue, liver, and colon were collected, frozen in liquid, nitrogen and stored at $-80^{\circ} \mathrm{C}$. A section of the main liver lobe was fixed-frozen in embedding medium (Tissue-tek, Sakura, the Netherlands), a piece of liver was fixed in formalin, and a piece of colon was fixed in Carnoy's solution or formalin for further histological analysis.

\subsection{Gut microbiota analyses}

Genomic DNA was extracted from the cecal content using a QIAamp DNA Stool Mini Kit (Qiagen, Hilden, Germany) according to the manufacturer's instructions, including a bead-beating step. 16S rDNA pyrosequencing and quantitative PCR (Qpcr) were performed as described previously and in Supporting Information Material and Methods 2 [19].

\subsection{Biochemical analysis}

Triglycerides and cholesterol were measured in the liver tissue after extraction with chloroform-methanol as described by Neyrinck et al. [20]. Lipid degradation products ensuing from oxidative stress in the liver were determined by measuring the concentration of thiobarbituric acid reactive substances (TBARS). Briefly, tissues were homogenized in saline using Tissue Lyser II (Qiagen, France). Aldehydes present in tissue lysate reacted with thiobarbituric acid forming an aldehyde-thiobarbituric acid complex, that was detected by spectrophotometry [21]. ROS were measured as described elsewhere [22]. Alanine aminotransferase (ALAT) levels were measured in the serum as markers of liver damage using the ALAT/GPT kit as described by the manufacturer (DiaSys Diagnostic and Systems).

\subsection{Histological analysis of the colon}

Crypt depth was measured after hematoxilin/eosin or blue alcian staining. Whole-mount sections were digitized using a whole-slide scanner (Leica SCN400, Leica Microsystems, Germany) and images were captured using the Leica Image Viewer Software (Version 4.0.4). Measurements of crypt depth were made on colon sections of at least ten crypts per animal with a method previously described [23].

\subsection{Tissue mRNA analyses}

Total RNA was isolated from tissues using the TriPure isolation reagent kit (Roche Diagnostics, Penzberg, Germany). RNA quality was checked using an Agilent 2100 Bioanalyzer (Agilent Technologies, Santa Clara, CA) with a quality threshold at 6 . Complementary DNA was prepared by reverse transcription of $1 \mu \mathrm{g}$ total RNA using the Kit Reverse Transcription System (Promega, Madison, WI). Real-time PCR was performed with a StepOnePlus Real-Time PCR System and software (Applied Biosystems, Den Ijssel, the Netherlands) using SYBR Green (Eurogentec, Seraing, Belgium) for detection. All samples were run in duplicate in a single 96-well reaction plate, and data were analyzed according to the $2^{-\Delta \Delta C T}$ method. The purity of the amplified product was checked by analyzing the melting curve performed at the end of the amplification step. The ribosomal protein L19 (RPL19) gene was chosen as a reference gene. The primer sequences of the targeted genes are available on request (audrey.neyrink@uclouvain.be).

\subsection{Statistical analysis}

Results are presented as means with their standard errors, combining both experiments. Statistical analysis was performed by one-way analysis of variance (ANOVA) followed by post hoc Tukey's multiple comparison tests using the GraphPad Prism software (version 5.00, GraphPad Software, San Diego, California, USA). The results were considered statistically significant when $p$ value was $<0.05$.

\section{Results}

\subsection{Rhubarb extract induced drastic changes in microbial diversity and populations}

The differences within the intestinal microbial population structure between the groups were visualized by non metric dimensional scaling built upon a distance matrix based on the species taxonomic level (Fig. 1A). A distinct cluster was observed for mice receiving rhubarb extract supplementation whereas the acute alcohol administration did not induce a major shift in the gut microbiota composition. In fact, alcohol administration did not change cecal microbial $\alpha$-diversity (Fig. 1B-D) and the relative abundance of bacteria at the phylum level (Fig. 2A and Supporting Information Table 3). However, we observed significant changes in the relative abundance of some species, such as higher proportion of Rikenellaceae (due to species belonging to Alistipes genus) and lower relative abundance of Verrucomicrobiaceae (due to Akkermansia muciniphila) and vadinBB60 group in the alcohol-alone treated mice versus the control one. In contrast, the rhubarb-treated mice exhibited a decreased cecal microbial $\alpha$-diversity compared to the control mice due to a lower evenness without affecting significantly the species richness (Fig. 1B-D). When comparing the relative abundance of bacterial taxa between treatment groups, we observed a significant phylum-wide shift from Firmicutes to Verrucomicrobia upon rhubarb extract supplementation (Fig. 2A and Supporting Information Table 3). At the family level, the abundance of Verrucomicrobiaceae significantly decreased due to the alcohol challenge. This effect was counteracted upon treatment with rhubarb extract and this population reached even $39 \%$ 
A

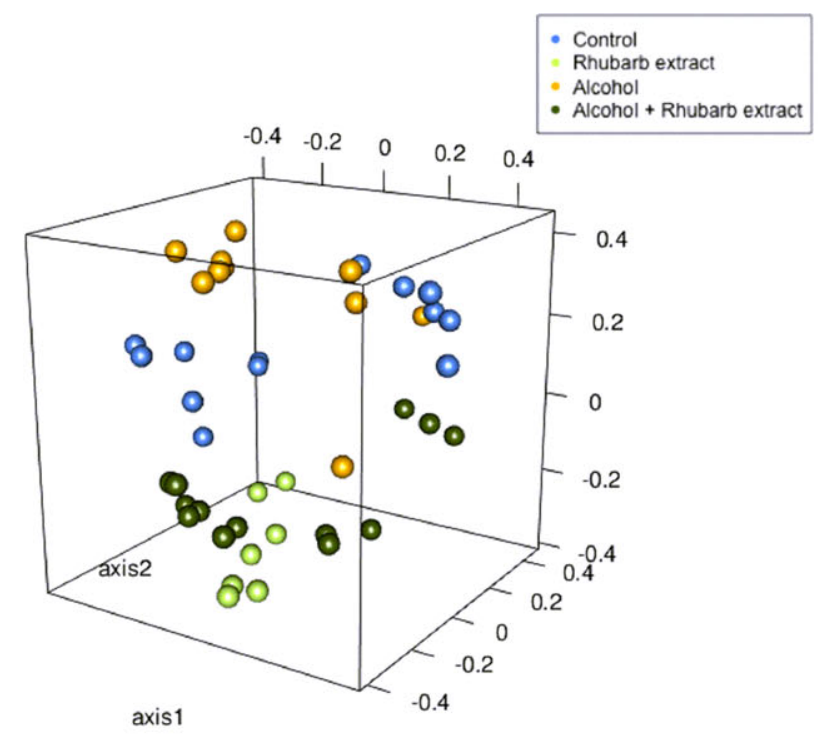

C
B

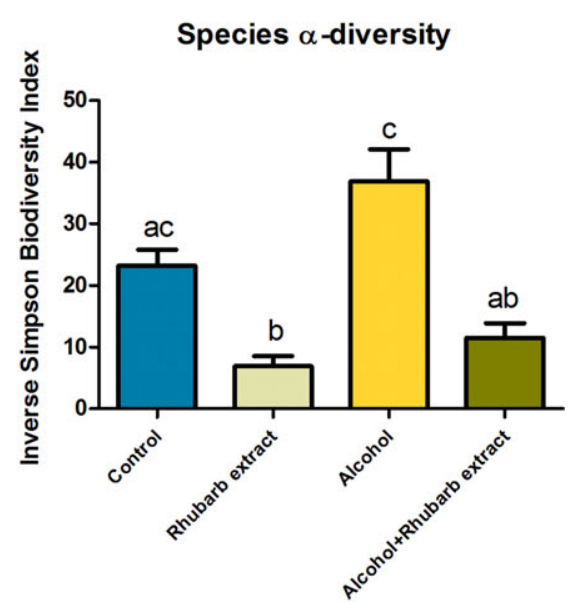

Species evenness

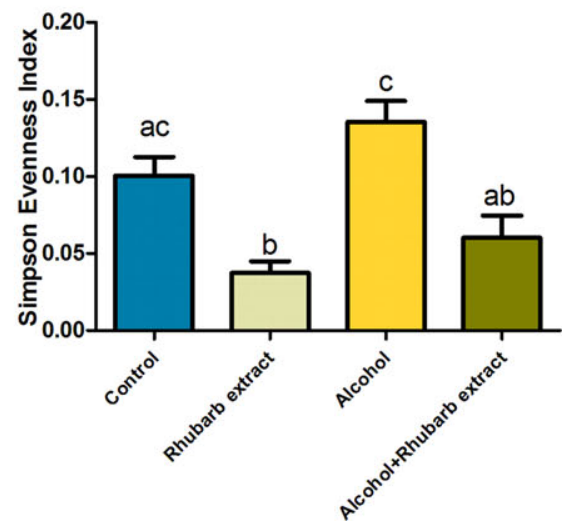

Figure 1. Spatial ordination and bacterial diversity deduced by $16 \mathrm{~S}$ profiling. (A) Nonmetric dimensional scaling with three axis of the four mice groups; (B) bacterial diversity (Inverse Simpson Biodiversity Index); (C) bacterial richness (Chao1 Richness Index); (D) bacterial evenness (deduced from Simpson Index). Rhubarb extract (light green), Alcohol (yellow), and Alcohol + Rhubarb extract (dark green). Mice were fed a control diet supplemented with or without rhubarb extract during 17 days before the alcohol challenge. Diversity indexes are expressed as the mean from subsampled datasets \pm SEM. Data with different superscript letters are significantly different at $p<0.05$ according to the one-way analysis of variance statistical analysis followed by Tukey post hoc test.

of abundance in the Rhubarb extract group. Other changes were observed after rhubarb extract supplementation such as a decrease in Lachnospiraceae, Prevotellaceae, as well as a modest decrease in vadin BB60 group. At a lower taxonomic level, the most prominent differences related to the Akkermansia genus whose relative abundance reached $40 \%$ in rhubarb extract-fed mice after the alcohol challenge, to the Alistipes genus whose relative abundance was increased by the alcohol challenge and lowered by rhubarb extract and to the Parabacteroides genus that was promoted by rhubarb extract. At the OTU level, the most prominent increases after rhubarb supplementation treatment corresponded to A. muciniphila. The effect on this species was confirmed by qPCR (Fig. 2B). In accordance with the analysis at the genus level, Parabacteroides goldsteinii was higher upon rhubarb extract treatment; this increase was also confirmed by qPCR analysis (Fig. 2C).

\subsection{Rhubarb extract improved intestinal homeostasis}

Rhubarb extract increased the number of feces produced over $24 \mathrm{~h}$ after $2 \mathrm{wk}$ of rhubarb treatment (Supporting Information Fig. 4). No diarrhea was observed (Supporting Information 

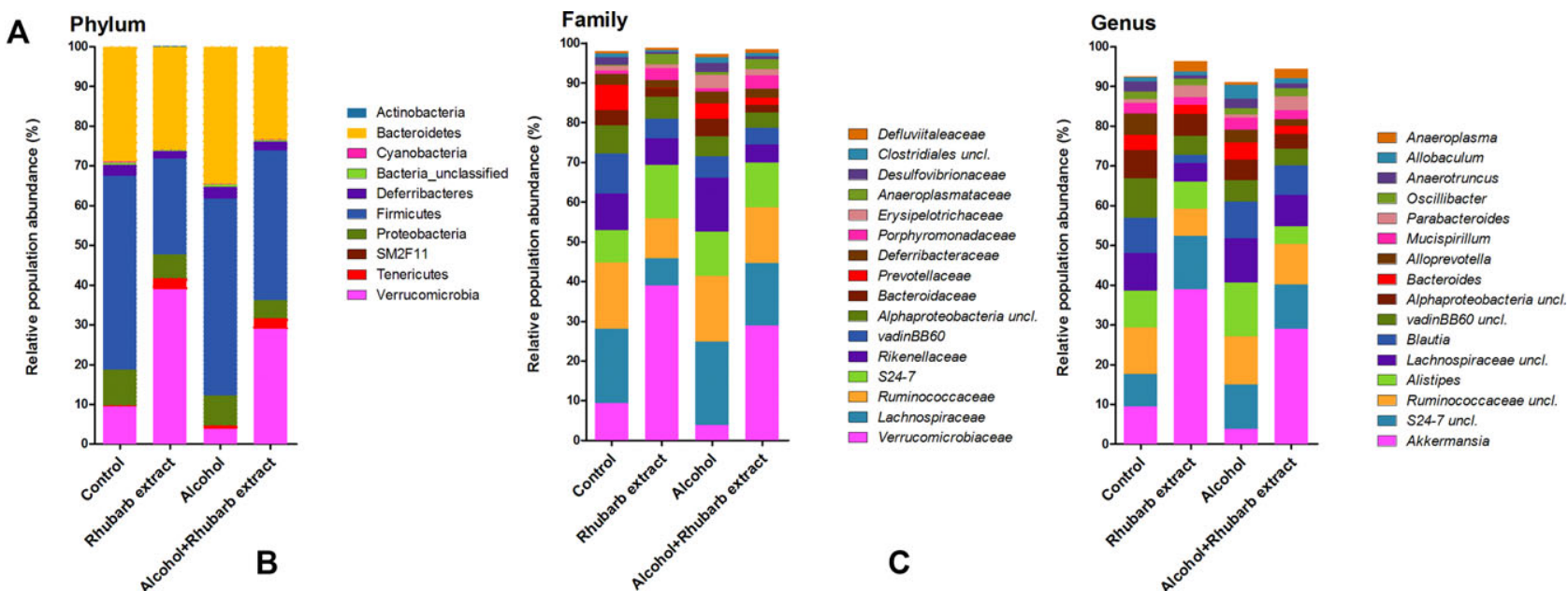

Akkermansia muciniphila
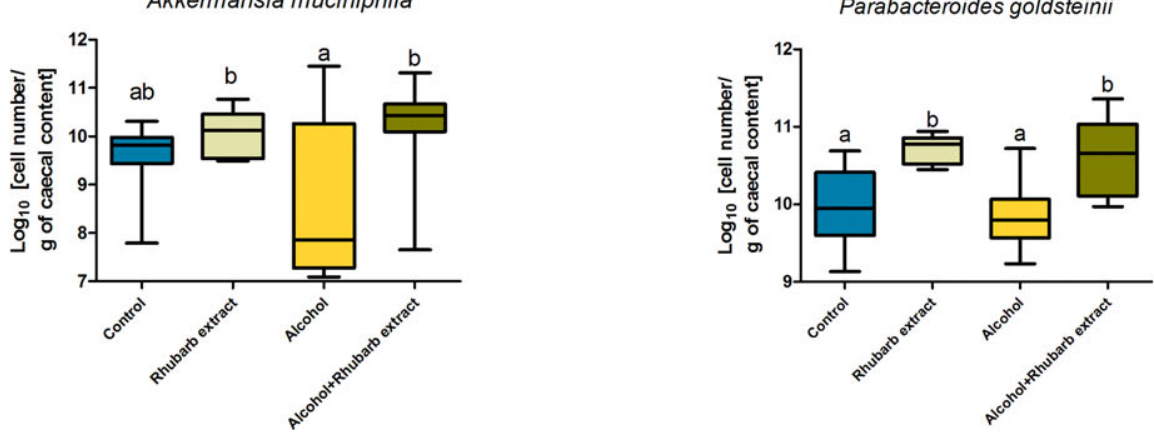

Figure 2. Changes in microbial populations in the cecal content, assessed by 454-pyrosequencing. (A) Relative abundances of bacterial taxa accounting for more than $1 \%$, at the phylum, family, and genus levels. (B) qPCR analysis of $A$. muciniphila in the cecal content. (C) qPCR analysis of $P$. goldsteinii in the cecal content. Mice were fed a control diet supplemented with or without rhubarb extract during 17 days before the alcohol challenge. Data are Whiskers plots with minimum and maximum for $\mathrm{B}$ and $\mathrm{C}$. Data with different superscript letters are significantly different at $p<0.05$ according to the one-way analysis of variance statistical analysis followed by Tukey post hoc test.

Fig. 4A). After 17 days of treatment, rhubarb extract enlarged colon tissue and increased cecal tissue weight and colon crypt depth independently of alcohol challenge (Fig. 3A-C). Moreover, rhubarb extract increased the mRNA expression of intectin by almost twofold in the absence of alcohol (Fig. 3D), suggesting a higher epithelial cell turnover [24, 25]; however this result did not reach statistical significance. Inflammatory markers measured in the colon were not significantly changed by rhubarb extract in comparison to their respective controls (Table 1).

Antimicrobial peptides are produced by the host and contribute to shape the composition of the gut microbiota. These peptides participate to the gut barrier function and are an attractive mechanism for the modulation of the gut ecosystem by nutrients. We measured the expression of secreting antimicrobial peptides produced by epithelial cells in the colon: lysozyme C (Lyz1), phospholipase A2 groupII (PlA2g2), $\alpha$-defensins (Defa), and C-type lectin, primarily the regenerating islet-derived 3-gamma (RegIII $\gamma)$. None of these antimicrobial peptides were significantly affected by the alcohol challenge (Fig. 4). Interestingly, rhubarb extract upregulated Pla2g2 and RegIII $\gamma$ expression, suggesting that rhubarb extract improved innate immunity through the secretion of antimicrobial peptides. Lys1 and Defa were not affected by the supplementation. Altogether, those results suggest that rhubarb extract improved intestinal homeostasis.

\subsection{Rhubarb extract improved alcohol-induced oxidative stress and inflammation in the liver}

As shown in Fig. 5, ethanol challenge induced the expression of several proinflammatory markers in the liver tissue. The administration of rhubarb extract reduced the expression of key inflammatory genes (TLR4, MCP-1, TNF- $\alpha$, IL6) and macrophage-related markers (F4/80, CD68, CD11c). Those data are in accordance with the detection and semiquantification of F4/80 by immunohistochemistry (Supporting Information Fig. 5A). Hepatic steatosis was examined using histological analysis after oil red O staining (Supporting Information Fig. 5B). Alcohol-exposed mice exhibited only a slight increase of fat accumulation. In fact, acute alcohol intake did not significantly induce triglyceride or 
A
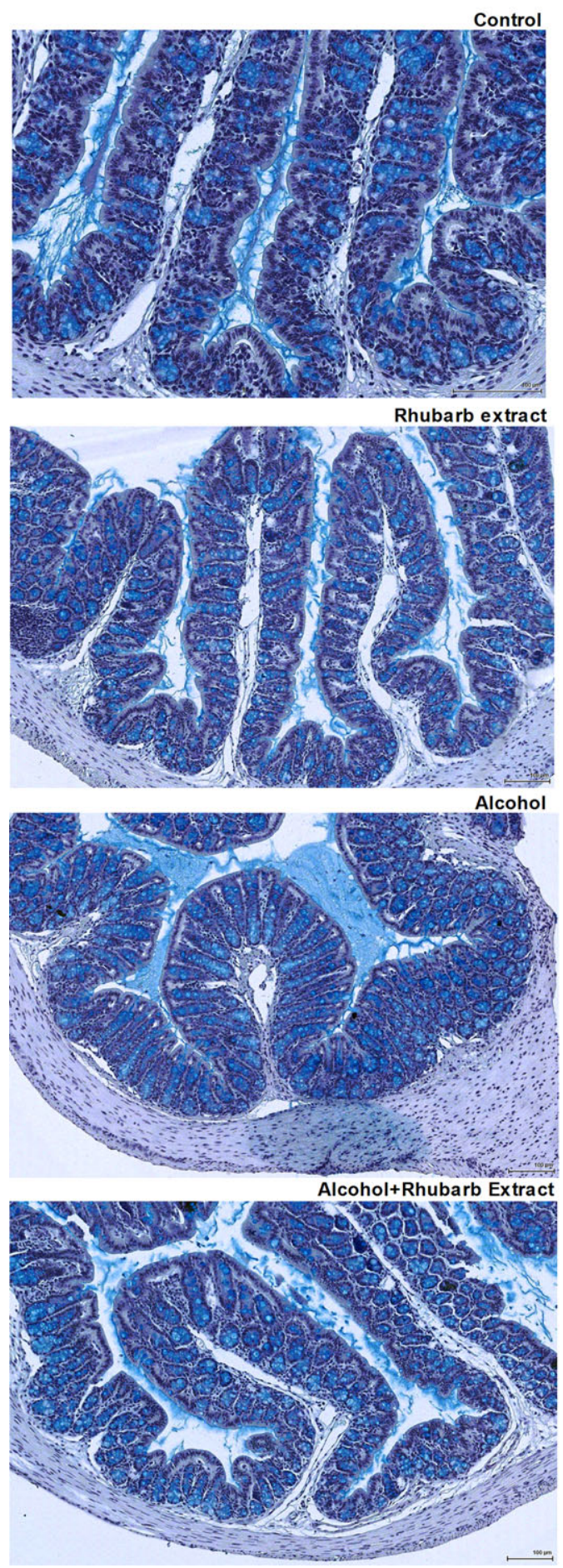

B

caecal tissue weight

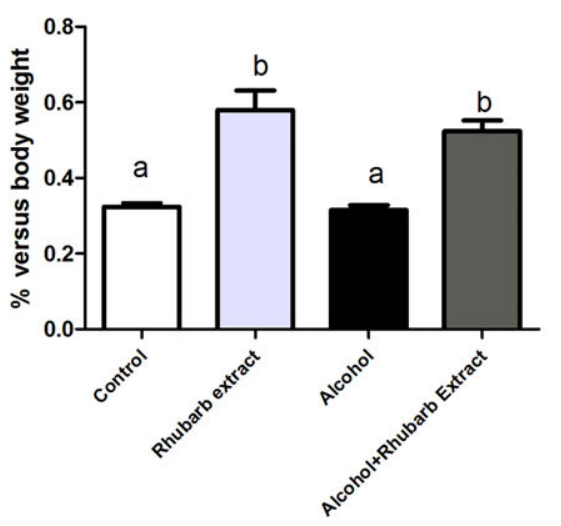

C

colon crypt depth

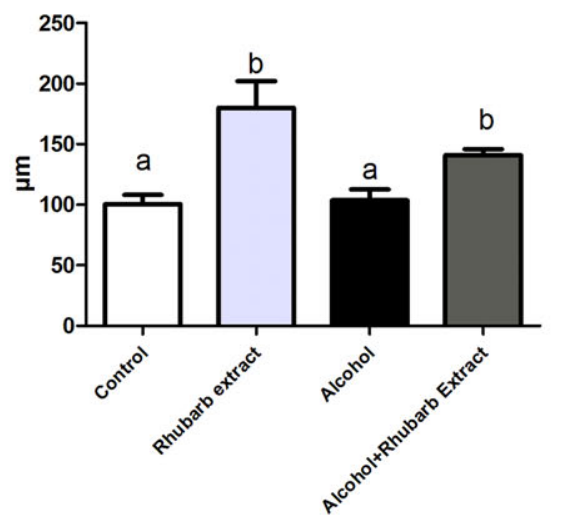

D

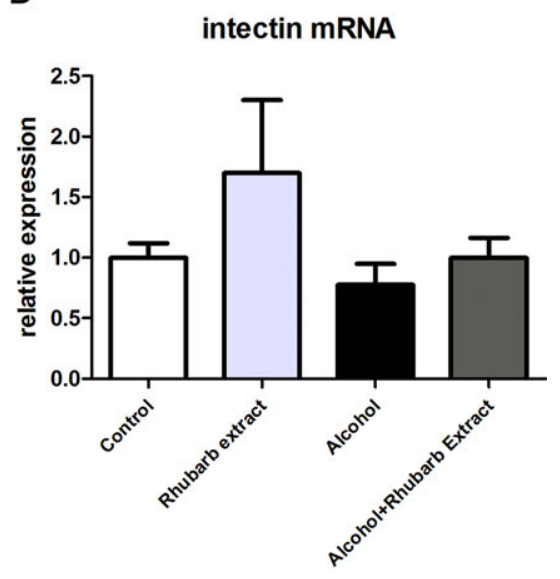

Figure 3. Morphologic, morphometric, and molecular changes for the evaluation of cell proliferation in the caecocolon. (A) Representative pictures of the colon after alcian blue staining; (B) cecal tissue weight versus body weight; (C) crypt depth measured by histological analyses after hematoxilin/eosin or blue alcian staining; (D) expression of intectin in the colon. Mice were fed a control diet supplemented with or without rhubarb extract during 17 days before the alcohol challenge. Data are expressed as the mean \pm SEM. Data with different superscript letters are significantly different at $p<0.05$ according to the one-way analysis of variance statistical analysis followed by Tukey post hoc test. cholesterol accumulation in the liver (Table 2). The supplementation with rhubarb extract tended to lessen lipid accumulation but this result did not reach statistical significance after alcohol intoxication (Table 2 and Supporting Information Fig. 5B).
We evaluated hepatic oxidative stress by measuring TBARS and ROS contents, as well as the gene expression of NADPH oxidase in the liver tissue. Supplementation with rhubarb extract to mice facing an alcohol challenge was able to decrease ROS production and the mRNA levels of NADPH 
Table 1. Gene expression in the colon

\begin{tabular}{|c|c|c|c|c|}
\hline mRNA (relative expression) & Control & $\begin{array}{l}\text { Rhubarb } \\
\text { extract }\end{array}$ & Alcohol & $\begin{array}{l}\text { Alcohol+ } \\
\text { Rhubarb extract }\end{array}$ \\
\hline II-6 & $1.00 \pm 0.08$ & $1.20 \pm 0.19$ & $0.94 \pm 0.17$ & $0.79 \pm 0.23$ \\
\hline II-1 $\beta$ & $1.00 \pm 0.09^{a}$ & $1.10 \pm 0.16^{a, b}$ & $1.37 \pm 0.21^{a, b}$ & $1.96 \pm 0.37^{b}$ \\
\hline TNF- $\alpha$ & $1.00 \pm 0.11$ & $1.17 \pm 0.23$ & $1.16 \pm 0.39$ & $1.27 \pm 0.16$ \\
\hline MCP-1 & $1.00 \pm 0.10^{a, b}$ & $1.32 \pm 0.26^{a}$ & $0.57 \pm 0.07^{b}$ & $1.07 \pm 0.20^{a, b}$ \\
\hline
\end{tabular}

Mice were fed a control diet supplemented with or without rhubarb extract during 17 days before the alcohol challenge. Data are expressed as the mean \pm SEM. Data with different superscript letters are significantly different at $p<0.05$ according to the one-way analysis of variance statistical analysis followed by Tukey post hoc test. MCP-1, monocyte chemotactic protein 1.

oxidase in the liver, while the hepatic levels of TBARS, ROS, and NADPH oxidase mRNA were not significantly affected by the ethanol administration (Table 2). Of note, rhubarb extract did not modify any of those parameters in the control condition (Fig. 5 and Table 2). Acute ethanol exposure caused also a higher release of ALAT in the serum compared with control animals. Mice pretreated with rhubarb extract exhibited lower level of ALAT in both control and alcohol conditions (ALAT activity expressed in U/L: $15.9 \pm 2.7,10.4 \pm 1.1,56.5 \pm 30.7$, $29.7 \pm 6.3 \mathrm{U} / \mathrm{L}$ for control, Rhubarb extract, Alcohol, and Alcohol + Rhubarb extract conditions, respectively); however, this effect did not reach statistical significance.
A
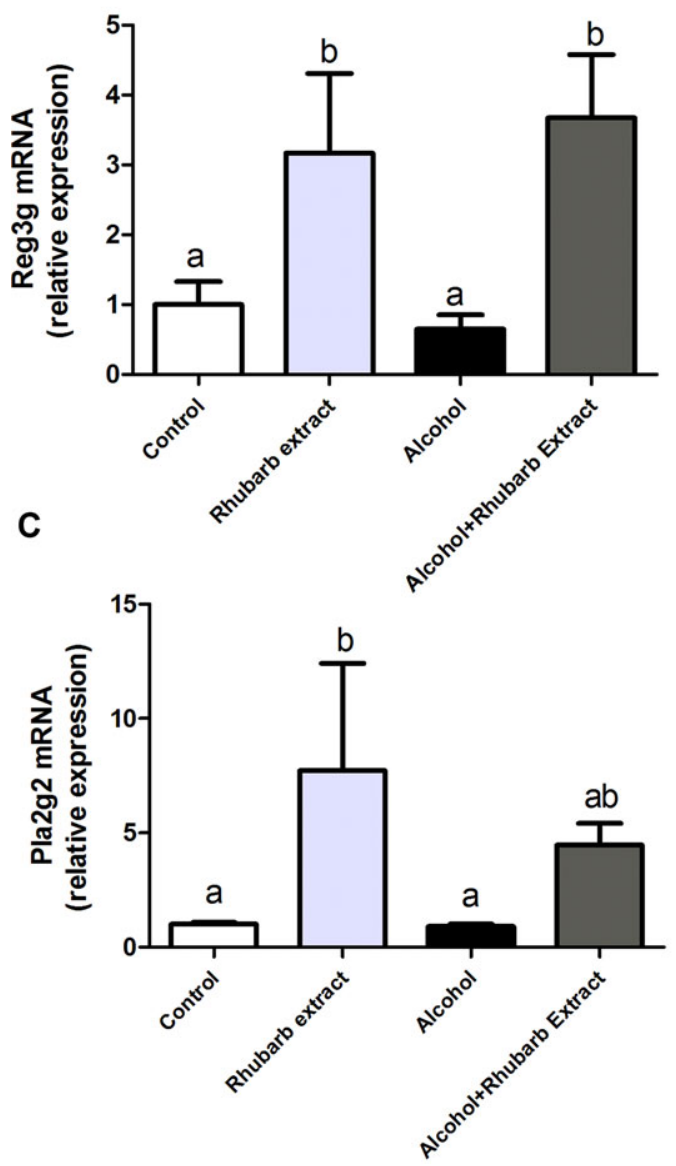

B
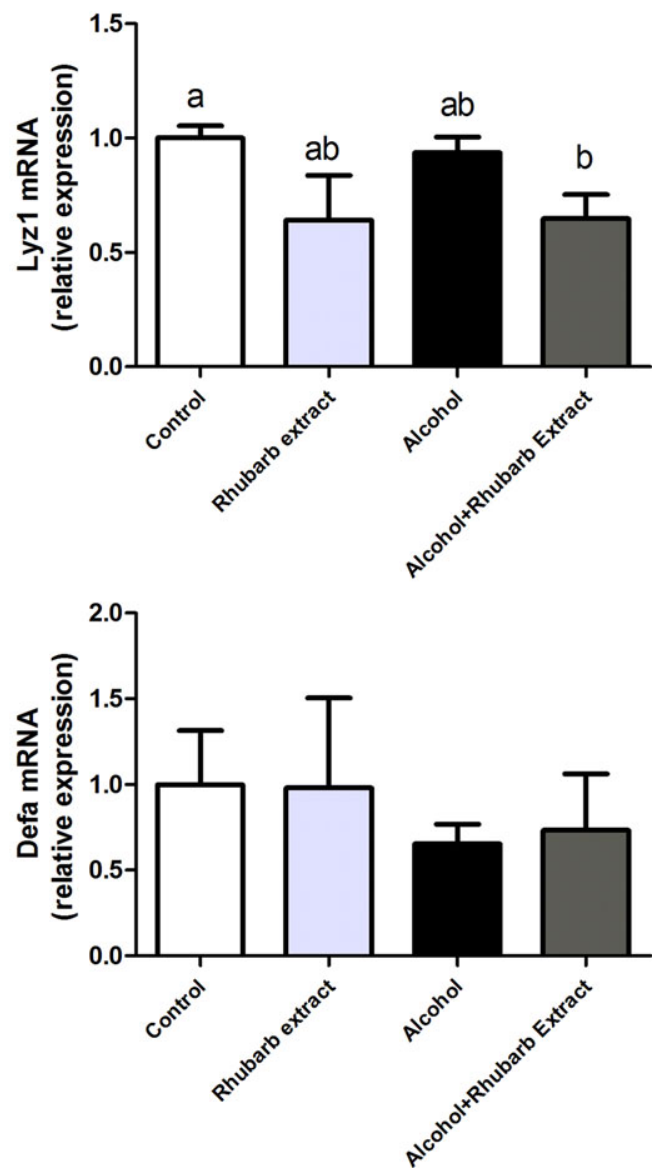

Figure 4. Expression of antibacterial peptides in the colon. (A) Regllly (encoded by Reg3g); (B) lysozyme C (encoded by Lyz1); (C) phospholipase A2 group II (encoded by Pla2g2); (D) $\alpha$-defensins (encoded by Defa). Mice were fed a control diet supplemented with or without rhubarb extract during 17 days before the alcohol challenge. Data are expressed as the mean $\pm \mathrm{SEM}$. Data with different superscript letters are significantly different at $p<0.05$ according to the one-way analysis of variance statistical analysis followed by Tukey post hoc test. 


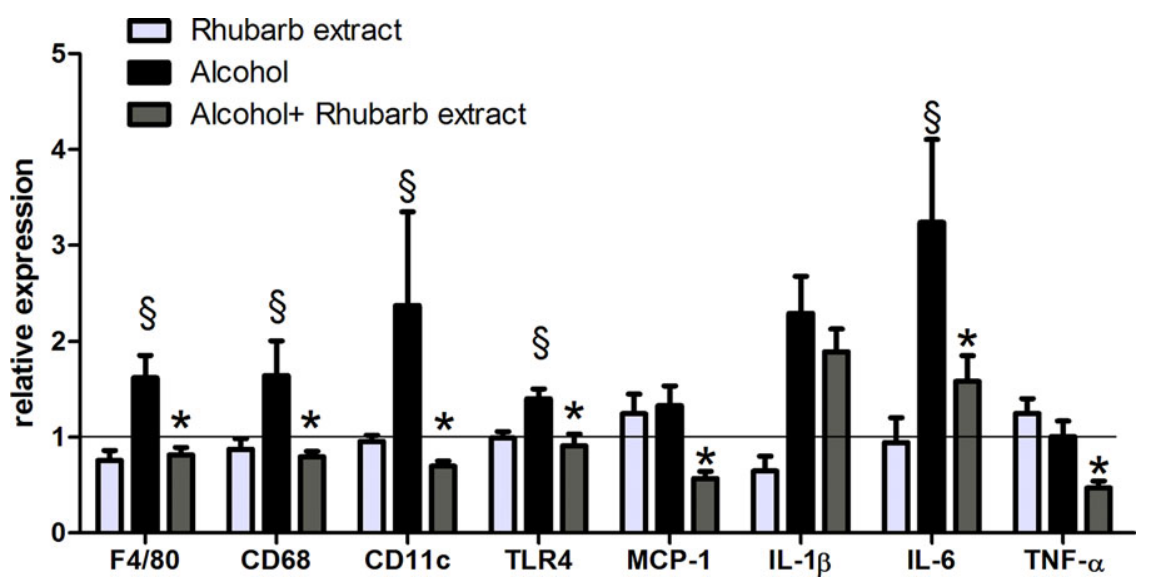

Figure 5. Expression of inflammatory genes in the liver. Data are expressed as the mean $\pm \mathrm{SEM}$; values are expressed relative to control group (set at 1 ). ${ }^{\S} p<0.05$ versus control group and ${ }^{*} p<0.05$ versus Alcohol group according to the one-way analysis of variance statistical analysis followed by Tukey post hoc test. Mice were fed a control diet supplemented with or without rhubarb extract during 17 days before the alcohol challenge.

\subsection{Changes in the gut microbiota composition induced by rhubarb extract correlated with host parameters}

We performed a Pearson correlation analysis adjusted for multiple testing according to the Bonferonni and Hochberg procedure in order to evaluate the potential links between significant changes in the gut microbiota composition induced by rhubarb extract and host metabolism and immunity (Fig. 6). We found that several gut bacteria were positively or negatively associated with hepatic inflammatory markers or gut antimicrobial peptides. For instance, we found that both RegIII $\gamma$ expression and colon crypt depth were positively associated with Verrucomicrobia, Verrucomicrobiaceae, Akkermansia, and finally A. muciniphila whereas P. goldsteinii from the Porphyromonadaceae family was negatively correlated with TNF- $\alpha$ expression in the liver. It is important to note that some species belonging to the Alistipes genus and Allobaculum genus or the families S24-7 group and Erysipelotrichaceae were positively correlated with hepatic inflammatory or oxidative stress markers.

\section{Discussion}

A prominent feature of alcohol abuse is disruption of the intestinal barrier. Animal models of ALD exhibit a leaky gut
[26], and impairments of the intestinal barrier function were found in an alcohol-dependent population [12]. Although endotoxemia ensuing from this intestinal barrier dysfunction is widely considered a major cause of liver injury and inflammatory disorders due to alcohol exposure, the exact mechanisms by which alcohol contribute to the altered intestinal permeability and endotoxemia are not fully understood. The integrity of the intestinal mucosa is determined by the function of several components: protective layer of defensins on the intraluminal surface of the intestinal epithelium; tight junction proteins between intestinal epithelial cells; and the gut immune cells in the intestinal wall [26]. Alcohol has both direct effects on these functions in the intestine and indirect effects by alcohol and/or its metabolites distributed via the blood stream [27-29]. Recently, the intestinal microbiome was evoked as a factor able to initiate and mediate an increase in intestinal permeability contributing to ALD $[8,10,11,30]$. Research focusing on the role of the microbiome in ALD is still in its infancy compared to other fields, such as obesity or fatty liver disease. Some authors reported that acute and moderate ethanol intake alters the epithelial barrier through ethanol oxidation into acetaldehyde by the colonic bacteria whereas others demonstrated that intestinal sterilization protects against alcohol-induced intestinal barrier leakage and prevents bacterial translocation [31,32]. In the present study, we observed that acute ethanol administration affected gut microbiota in the cecal content of mice. Proportions of

Table 2. Hepatic parameters related to steatosis and oxidative stress

\begin{tabular}{|c|c|c|c|c|}
\hline & Control & $\begin{array}{l}\text { Rhubarb } \\
\text { extract }\end{array}$ & Alcohol & $\begin{array}{l}\text { Alcohol+ } \\
\text { Rhubarb extract }\end{array}$ \\
\hline Triglycerides content (nmol mg protein) & $151 \pm 8^{a, b}$ & $118 \pm 9^{a}$ & $174 \pm 16^{\mathrm{b}}$ & $173 \pm 6^{b}$ \\
\hline Cholesterol content (nmol mg protein) & $81 \pm 6$ & $83 \pm 4$ & $98 \pm 9$ & $81 \pm 7$ \\
\hline TBARS content (mmol MDA/I homogenate $\mathrm{H} / 10$ ) & $5.2 \pm 0.4$ & $3.7 \pm 0.2$ & $5.1 \pm 0.7$ & $5.6 \pm 0.5$ \\
\hline 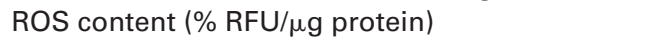 & $100 \pm 6^{a, b}$ & $84 \pm 13^{a, b}$ & $116 \pm 10^{a}$ & $85 \pm 6^{b}$ \\
\hline NADPH oxidase mRNA (relative expression) & $1.00 \pm 0.18^{a, b}$ & $0.87 \pm 0.23^{a, b}$ & $1.73 \pm 0.56^{a}$ & $0.55 \pm 0.06^{b}$ \\
\hline
\end{tabular}

Mice were fed a control diet supplemented with or without rhubarb extract during 17 days before the alcohol challenge. Data are expressed as the mean \pm SEM. Data with different superscript letters are significantly different at $p<0.05$ according to the one-way analysis of variance statistical analysis followed by Tukey post hoc test. MDA, relative fluorescence units; RFU, relative fluorescence units; ROS, reactive oxygen species; TBARS, thiobarbituric acid reactive substances. 

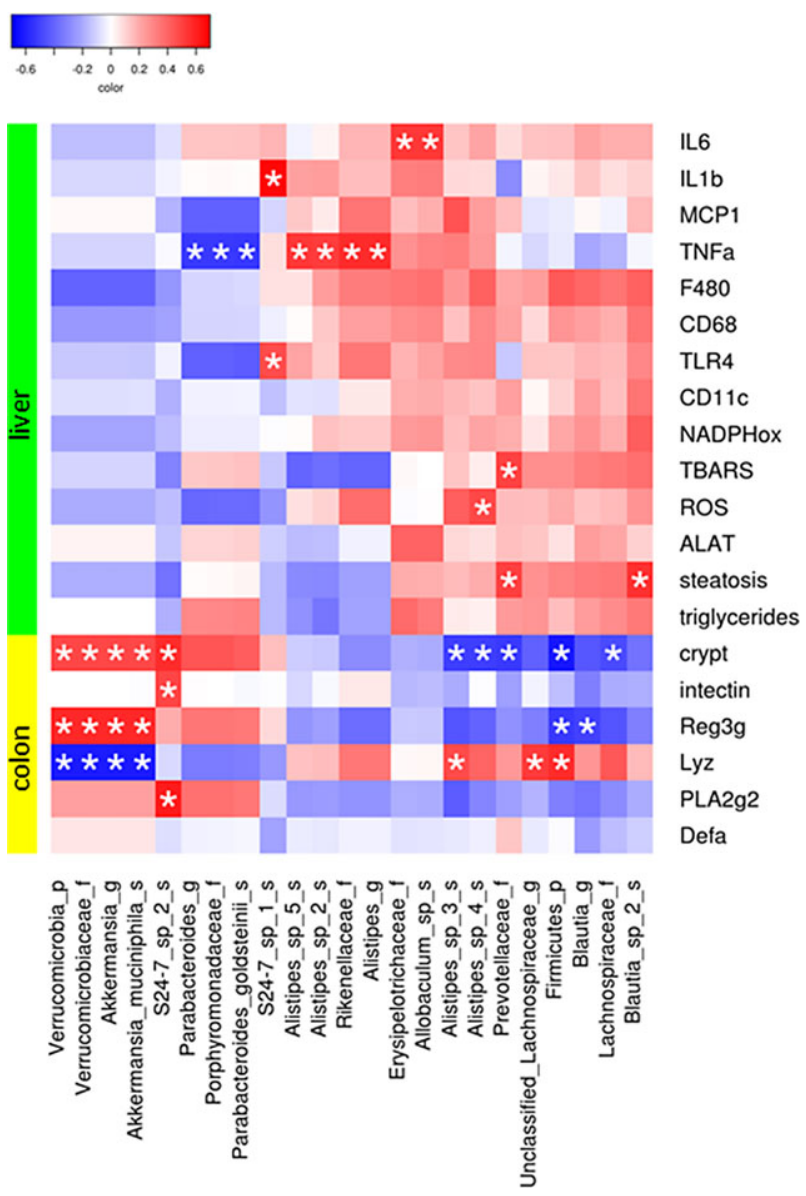

Figure 6. Correlations between bacterial taxa and host parameters. Pearson correlations were computed for all bacterial taxa significantly affected by the treatment (Supporting Information Table 3) and all measured host parameters. p-Values were adjusted for multiple testing according to the Bonferroni and Hochberg procedure. The color at each intersection refers to the value of the $r$ coefficient; asterisk indicated a significance correlation between these two parameters $(p<0.05)$. Only the bacterial taxa for which at least one significant correlation with a host parameter was detected, are displayed. Bacterial taxonomic level is indicated at the end of the name $(p=$ phylum, $f=$ family, $g=$ genus, $\mathrm{s}=$ species).

Verrucomicrobiaceae, in particular A. muciniphila population, disappeared from our targeted metagenomic analysis whereas the abundance of Alistipes was higher $6 \mathrm{~h}$ after alcohol administration. It is worth noting that data presented herein demonstrated an important modulation of the gut microbiota in favor of $A$. muciniphila and $P$. goldsteinii by the rhubarb extract per se, independently of alcohol administration. We cannot exclude that the modulation of gut microbiota was linked to the carbohydrate or fiber contents in the rhubarb extract but their concentrations in the diet $(0.21$ and $0.08 \%$, respectively) were probably too low to induce such an effect. Indeed, prebiotic effect of inulin-type fructans was generally observed at doses higher than $1 \%$ in the diet of ani- mals [33]. Polyphenols at low doses influence gut microbiota composition (for review [34,35]). Therefore, we postulate that changes of gut microbiota observed in our study could be induced by the most important antraquinone present in the rhubarb extract (rhein or its glucoside derivatives).

By which mechanisms would alcohol intake alter the composition of the intestinal microbiota? Multiple factors are likely to contribute to changes in the intestinal microbiome during development of ALD, and one of them is the intestinal innate immune system. Antimicrobial molecules, which are part of the innate immune response, are secreted from epithelial cells. In particular, the antimicrobial molecules RegIII $\gamma$ are reduced in the small intestines of mice following $3 \mathrm{wk}$ of intragastric ethanol feeding [36]. In the present study, acute alcohol intake did not alter the levels of expression of these antimicrobial proteins. However, RegIII $\gamma$ and Pla2g2 were increased by the rhubarb extract. This suggests that, under rhubarb extract supplementation, the host contributes to the modification of the microbial community by modulating the production of specific antimicrobial peptides [37, 38].

Moreover, rhubarb supplementation increased crypt depth and intestinal tissue weight. Consistent with this observation, we found that the rhubarb extract tended to increase the mRNA expression of intectin in absence of alcohol administration. In fact, intectin is a small intestine-specific glycosylphosphatidylinositol-anchored protein that has been proposed to be involved in the rapid turnover of intestinal mucosa [24]. These findings suggest that rhubarb extract increases epithelial cell turnover that could constitute a new mechanism contributing to reinforce the intestinal barrier, as suggested for inulin-type fructans [25, 38, 39].

If controversies exist concerning the potential beneficial or harmful effect on health of rhubarb herb and its main constituents (anthraquinones like rhein, emodin, and aloeemodin), some human intervention studies devoted to evaluate the effect of rhubarb supplementation on chronic kidney diseases or menopausal symptoms were reported to be safe $[40,41]$. In rodents, several in vivo experiments demonstrated hepatoprotective effects of rhubarb and its anthraquinone compounds [42-44]. Anyway, two cases of rhubarb-induced hepatotoxicity have been reported in animals $[45,46]$. In these both last studies, the doses of rhubarb extract were 3 to $20 \mathrm{~g} / \mathrm{kg}$ of body weight per day and were equivalent to 6 to 40 times of the upper dose of human stipulated in Chinese Pharmacopoeia $(0.5 \mathrm{~g} / \mathrm{kg})$, respectively. In our study, we decided to work with more realistic doses as compared to the human use: the dose of $0.3 \%$ in the diet corresponded to a maximum of $0.35 \mathrm{~g} / \mathrm{kg}$ per day. This dose did not induce hepatic alterations: we have shown that transaminase (ALAT) activity in the serum tended to decrease upon rhubarb supplementation, suggesting no hepatic injury due to rhubarb extract, independently of alcohol challenge.

Oxidative stress (coming from the ROS overproduction by parenchymal and nonparenchymal cells) is also considered as a well-known mediator in the development of liver damage [47]. Importantly, although ROS levels were not 
significantly increased due to alcohol challenge as compared to control conditions, we observed that the rhubarb extract had clear antioxidant effects as shown by the significant reduction in hepatic ROS levels in Alcohol + Rhubarb extract treated mice. ROS could be produced by hepatocytes through cytochrome P450E1 system [48]. However, when alcohol is consumed, the most likely source of free radicals seems to be the ROS derived from NADPH oxidase in activated Kupffer cells [49]. The most compelling evidence corroborating the role of oxidative stress in ALD was reported from study performed with NADPH oxidase (PHOX-47) knockout mice, which were found to be resistant to hepatotoxicity induced by chronic alcohol consumption [49]. Interestingly, rhubarb extract blunted the ethanol-induced NADPH oxidase expression in our binge drinking model. Nonetheless, no antiperoxidative activity of the natural extract could be evidenced through TBARS assay.

It is well known that acute and chronic alcohol exposure induces translocation of bacterial components and primes their binding to TLR4 on the surface of hepatic Kupffer cells, thereby leading to the activation of NF-kB-mediated TNF$\alpha$ signaling and resulting in inflammation $[1,13,50,51]$. In the present study, several inflammatory markers were induced by acute alcohol challenge. We also observed an infiltration of macrophages inside the liver tissue by using both molecular and histological analysis. Interestingly, rhubarb extract blunted hepatic inflammation induced by acute ethanol treatment. Indeed, our data showed that rhubarb extract suppressed significantly the expression of IL-6, MCP-1, and TNF- $\alpha$ in mice facing an acute alcohol challenge. Moreover, macrophage infiltration in the liver was normalized as shown by the significant downregulation found in the expression levels of F4/80 (confirmed by histological analysis) and other markers of macrophage such as CD68 and CD11c. Importantly, supplementation with rhubarb extract was found to inhibit also the expression of TLR4 in the liver. In fact, alteration of TLR signaling is one of the major mechanisms involved in the impairment of immune response because TLR serve as pattern recognition receptors on macrophages and other innate immune cell types [52]. Consequently, we would suggest an inhibition of the TLR4-dependent inflammation in liver tissue after rhubarb extract supplementation, which would reflect an enhancement of the hepatic immune response.

Interestingly, the effect of rhubarb extract on inflammation and oxidative stress in the liver tissue may occur independently of any significant changes in lipid accumulation. This led us to postulate that the management of hepatic inflammation or oxidative stress could be due to other mechanisms, and we have tested the hypothesis of the involvement of the gut (barrier) function and microbiota. The use of two independent $16 \mathrm{~S}$ rRNA gene-based techniques revealed that the relative abundance of the bacterium A. muciniphila was increased upon rhubarb supplementation. As A. muciniphila abundance was positively correlated with the colonic expression of RegIII $\gamma$, one could hypothesize that rhubarb extract modules the colonic expression of RegIII $\gamma$ by promoting the growth of A. muciniphila. These results are in accordance with previous studies showing that treatments that change the gut microbiota in favor of $A$. muciniphila restore gut barrier function and increases RegIII $\gamma$ mRNA expression [38, 53]. In accordance with this hypothesis, it was recently demonstrated that A. muciniphila decreased dramatically in genetically and diet-induced obese mice. Furthermore, prebiotic (oligofructose) treatment that restored A. muciniphila abundance and oral administration of this bacterium improved gut barrier, metabolic parameters, and inflammatory disorders $[38,53,54]$. The physiological role of $P$. goldsteinii is much less understood. Administration of a mushroom extract ( $\mathrm{Gan}$ oderma lucidum) to high fat-fed mice led to an increased relative abundance of $P$. goldsteinii, from 3 to $25 \%$, and this was associated with a leaner phenotype, decreased activation of the TLR4 pathway in the liver, and reduced hepatic inflammation $[55,56]$. In our study, $P$. goldsteinii was negatively correlated with one important marker involved in TLR4 pathway (TNF- $\alpha$ ).

In summary, this study highlighted that administration of the rhubarb extract at low doses modified host antimicrobial peptide production and gut homeostasis and was associated with profound changes in gut microbial composition. Moreover, the administration of the rhubarb extract had a hepatoprotective effect in binge alcohol induced liver injury acting upon the first step of the disease. This outcome might be the consequence of several pathways related with the downregulation of expression levels of inflammatory and oxidative markers such as TLR4 and NADPH oxidase, respectively. We hypothesize that the changes in gut bacteria observed upon rhubarb treatment was involved in the higher turnover of epithelial cells contributing to reinforce gut barrier, and thereby, would reduce hepatic damages induced by a binge alcoholic challenge. Future studies should aim at further unraveling the mechanisms by which the gut microbiota impact host physiology related to alcohol abuse, with the hypothesis in mind that the gut microbiota could either be a contribution factor and a therapeutic target in this context.

We thank Remi Selleslagh, Isabelle Blave, and Bouazza Es Saadi for skillful technical assistance and Carine Nezer, Quality Partner s.a., Herstal, Belgium, for performing metagenetic analysis. L.B.B. and A.E. are Postdoctoral Researchers from the FRSFNRS (Fond National de la Recherche Scientifique, Belgium). N.M.D. is a recipient of FRS-FNRS grants (CDR J.0122.15). N.M.D. and G.D. are recipients of grant from the Wallonia supported by the competitive cluster Wagralim (NUTRIGUTIOR project, convention 6918). P.D.C., a research associate at the FRSFNRS, Belgium, is a recipient of an ERC Starting Grant 2013 (European Research Council, Starting grant 336452-ENIGMO), PDR subsidies (Projet de recherches T0.138.14; Fonds de la recherche scientifique, Belgium), and ARC (Concerted Research Activities-French Community of Belgium convention: 12/17-047) and is supported by the FRS-FNRS via the FRFS-WELBIO under Grant number WELBIO-CR-2012S-02R. The doctoral fellowship 
of UE is financed by the Department of Education, Language Policy and Culture from Government of the Basque Country.

A.M.N. and N.M.D. conceived and designed the experiments. A.M.N. and U.E. performed the experiments, data analysis, and interpretation. B.T. performed pyrosequencing and analyzed the gut microbiome data. M.V.H. performed histological analysis. L.B.B. performed correlation analysis. A.M.N., U.E., and N.M.D. wrote the manuscript. P.D.C., L.B.B, A.E., and G.D. provided intellectual input on the paper and reviewed the paper. N.M.D. planned and supervised all experiments and manuscript preparation. All of the authors read and approved the final manuscript.

The authors have declared no conflict of interest.

\section{References}

[1] Shukla, S. D., Pruett, S. B., Szabo, G., Arteel, G. E., Binge ethanol and liver: new molecular developments. Alcohol Clin. Exp. Res. 2013, 37, 550-557.

[2] Dugum, M., McCullough, A., Diagnosis and management of alcoholic liver disease. J. Clin. Transl. Hepatol. 2015, 3, 109116.

[3] Mantena, S. K., King, A. L., Andringa, K. K., Eccleston, H. B. et al., Mitochondrial dysfunction and oxidative stress in the pathogenesis of alcohol- and obesity-induced fatty liver diseases. Free Radic. Biol. Med. 2008, 44, 1259-1272.

[4] Donohue, T. M. Jr., Osna, N. A., Trambly, C. S., Whitaker, N. P. et al., Early growth response- 1 contributes to steatosis development after acute ethanol administration. Alcohol Clin. Exp. Res. 2012, 36, 759-767.

[5] Donohue, T. M. Jr., Alcohol-induced steatosis in liver cells. World J. Gastroenterol. 2007, 13, 4974-4978.

[6] Stewart, S., Jones, D., Day, C. P., Alcoholic liver disease: new insights into mechanisms and preventative strategies. Trends Mol. Med. 2001, 7, 408-413.

[7] Marmier, S., Dentin, R., Daujat-Chavanieu, M., Guillou, H. et al., Novel role for carbohydrate responsive element binding protein in the control of ethanol metabolism and susceptibility to binge drinking. Hepatology 2015, 62, 1086-1100.

[8] Vassallo, G., Mirijello, A., Ferrulli, A., Antonelli, M. et al., Review article: alcohol and gut microbiota - the possible role of gut microbiota modulation in the treatment of alcoholic liver disease. Aliment. Pharmacol. Ther. 2015, 41, 917-927.

[9] Massey, V. L., Arteel, G. E., Acute alcohol-induced liver injury. Front. Physiol. 2012, 3, 193.

[10] Canesso, M. C. C., Lacerda N. L., Ferreira C. M., Gonçalves J. L. et al., Comparing the effects of acute alcohol consumption in germ-free and conventional mice: the role of the gut microbiota. BMC Microbiol. 2014, 14, 240.

[11] Hammer, A. M., Khan, O. M., Morris, N. L., Li, X. et al., The effects of alcohol intoxication and burn injury on the expression of claudins and mucins in the small and large intestines. Shock 2016, 45, 73-81.

[12] Leclercq, S., Matamoros, S., Cani, P. D., Neyrinck, A. M. et al., Intestinal permeability, gut-bacterial dysbiosis, and behavioral markers of alcohol-dependence severity. Proc. Natl. Acad. Sci. U S A 2014, 111, E4485-E4493.

[13] Gao, B., Bataller, R., Alcoholic liver disease: pathogenesis and new therapeutic targets. Gastroenterology 2011, 141, 1572-1585.

[14] Pan, M. H., Lai, C. S., Ho, C. T., Anti-inflammatory activity of natural dietary flavonoids. Food Funct. 2010, 1, 15-31.

[15] Yao, L. H., Jiang, Y. M., Shi, J., Tomas-Barberan, F. A. et al., Flavonoids in food and their health benefits. Plant Foods Hum. Nutr. 2004, 59, 113-122.

[16] Ding, R. B., Tian, K., Huang, L. L., He, C. W. et al., Herbal medicines for the prevention of alcoholic liver disease: a review. J. Ethnopharmacol. 2012, 144, 457-465.

[17] Huang, Q., Lu, G., Shen, H. M., Chung, M. C. et al., Anti-cancer properties of anthraquinones from rhubarb. Med. Res. Rev. 2007, 27, 609-630.

[18] Zhao, Y. L., Wang, J. B., Zhou, G. D., Shan, L. M. et al., Investigations of free anthraquinones from rhubarb against alphanaphthylisothiocyanate-induced cholestatic liver injury in rats. Basic Clin. Pharmacol. Toxicol. 2009, 104, 463-469.

[19] Bindels, L. B., Neyrinck, A. M., Salazar, N., Taminiau, B. et al., Non digestible oligosaccharides modulate the gut microbiota to control the development of leukemia and associated cachexia in mice. PLoS One 2015, 10, e0131009.

[20] Neyrinck, A. M., Possemiers, S., Verstraete, W., De, B. F. et al., Dietary modulation of clostridial cluster XIVa gut bacteria (Roseburia spp.) by chitin-glucan fiber improves host metabolic alterations induced by high-fat diet in mice. J. Nutr. Biochem. 2012, 23, 51-59.

[21] Esterbauer, H., Cheeseman, K. H., Determination of aldehydic lipid peroxidation products: malonaldehyde and 4hydroxynonenal. Methods Enzymol. 1990, 186, 407-421.

[22] Szabados, E., Fischer, G. M., Toth, K., Csete, B. et al., Role of reactive oxygen species and poly-ADP-ribose polymerase in the development of AZT-induced cardiomyopathy in rat. Free Radic. Biol. Med. 1999, 26, 309-317.

[23] De, C. C., Oevermann, A., Burgener, I. A., Doherr, M. G. et al., Gastrointestinal tract mucosal histomorphometry and epithelial cell proliferation and apoptosis in neonatal and adult dogs. J. Anim. Sci. 2010, 88, 2255-2264.

[24] Kitazawa, H., Nishihara, T., Nambu, T., Nishizawa, $\mathrm{H}$. et al., Intectin, a novel small intestine-specific glycosylphosphatidylinositol-anchored protein, accelerates apoptosis of intestinal epithelial cells. J. Biol. Chem. 2004, 279, 42867-42874.

[25] Cliffe, L. J., Humphreys, N. E., Lane, T. E., Potten, C. S. et al., Accelerated intestinal epithelial cell turnover: a new mechanism of parasite expulsion. Science 2005, 308, 1463-1465.

[26] Szabo, G., Gut-liver axis in alcoholic liver disease. Gastroenterology 2015, 148, 30-36.

[27] Rao, R. K., Acetaldehyde-induced barrier disruption and paracellular permeability in Caco-2 cell monolayer. Methods Mol. Biol. 2008, 447, 171-183.

[28] Wang, Y., Liu, Y., Sidhu, A., Ma, Z. et al., Lactobacillus rhamnosus GG culture supernatant ameliorates acute alcoholinduced intestinal permeability and liver injury. Am. J. Physiol Gastrointest. Liver Physiol. 2012, 303, G32-G41. 
[29] Turner, J. R., Intestinal mucosal barrier function in health and disease. Nat. Rev. Immunol. 2009, 9, 799-809.

[30] Schnabl, B., Brenner, D. A., Interactions between the intestinal microbiome and liver diseases. Gastroenterology 2014, 146, 1513-1524.

[31] Adachi, Y., Moore, L. E., Bradford, B. U., Gao, W. et al., Antibiotics prevent liver injury in rats following long-term exposure to ethanol. Gastroenterology 1995, 108, 218-224.

[32] Ferrier, L., Berard, F., Debrauwer, L., Chabo, C. et al., Impairment of the intestinal barrier by ethanol involves enteric microflora and mast cell activation in rodents. Am. J. Pathol. 2006, 168, 1148-1154.

[33] Roberfroid, M., Gibson, G. R., Hoyles, L., McCartney, A. L. et al., Prebiotic effects: metabolic and health benefits. Br. J. Nutr. 2010, 104 (Suppl 2), S1-63.

[34] Duenas, M., Munoz-Gonzalez, I., Cueva, C., Jimenez-Giron, A. et al., A survey of modulation of gut microbiota by dietary polyphenols. Biomed. Res. Int. 2015, 2015, 850902.

[35] Anhe, F. F., Varin, T. V., Le, B. M., Desjardins, Y. et al., Gut microbiota dysbiosis in obesity-linked metabolic diseases and prebiotic potential of polyphenol-rich extracts. Curr. Obes. Rep. 2015, 4, 389-400.

[36] Yan, A. W., Fouts, D. E., Brandl, J., Starkel, P. et al., Enteric dysbiosis associated with a mouse model of alcoholic liver disease. Hepatology 2011, 53, 96-105.

[37] Gallo, R. L., Hooper, L. V., Epithelial antimicrobial defence of the skin and intestine. Nat. Rev. Immunol. 2012, 12, 503516.

[38] Everard, A., Lazarevic, V., Gaia, N., Johansson, M. et al., Microbiome of prebiotic-treated mice reveals novel targets involved in host response during obesity. ISME J. 2014, 8, 2116-2130.

[39] Vereecke, L., Beyaert, R., van, L. G., Enterocyte death and intestinal barrier maintenance in homeostasis and disease. Trends Mol. Med. 2011, 17, 584-593.

[40] Khan, I. A., Nasiruddin, M., Haque, S. F., Khan, R. A., Evaluation of Rhubarb Supplementation in Stages 3 and 4 of Chronic Kidney Disease: A Randomized Clinical Trial. Int. J. Chronic Dis. 2014, 2014, 789340.

[41] Hasper, I., Ventskovskiy, B. M., Rettenberger, R., Heger, P. W. et al., Long-term efficacy and safety of the special extract $\mathrm{ERr} 731$ of Rheum rhaponticum in perimenopausal women with menopausal symptoms. Menopause 2009, 16, 117131.

[42] Ding, Y., Zhao, L., Mei, H., Zhang, S. L. et al., Exploration of Emodin to treat alpha-naphthylisothiocyanate-induced cholestatic hepatitis via anti-inflammatory pathway. Eur. J. Pharmacol. 2008, 590, 377-386.
[43] Arosio, B., Gagliano, N., Fusaro, L. M., Parmeggiani, L. et al., Aloe-emodin quinone pretreatment reduces acute liver injury induced by carbon tetrachloride. Pharmacol. Toxicol. 2000, 87, 229-233.

[44] Jin, H., Sakaida, I., Tsuchiya, M., Okita, K., Herbal medicine Rhei rhizome prevents liver fibrosis in rat liver cirrhosis induced by a choline-deficient L-amino acid-defined diet. Life Sci. 2005, 76, 2805-2816.

[45] Wang, J. B., Zhao, H. P., Zhao, Y. L., Jin, C. et al., Hepatotoxicity or hepatoprotection? Pattern recognition for the paradoxical effect of the Chinese herb Rheum palmatum $L$. in treating rat liver injury. PLoS One 2011, 6, e24498.

[46] Wang, J., Zhao, Y., Xiao, X., Li, H. et al., Assessment of the renal protection and hepatotoxicity of rhubarb extract in rats. J. Ethnopharmacol. 2009, 124, 18-25.

[47] Dryden, G. W. Jr., Deaciuc, I., Arteel, G., McClain, C. J., Clinical implications of oxidative stress and antioxidant therapy. Curr. Gastroenterol. Rep. 2005, 7, 308-316.

[48] Morgan, K., French, S. W., Morgan, T. R., Production of a cytochrome P450 2E1 transgenic mouse and initial evaluation of alcoholic liver damage. Hepatology 2002, 36, 122-134.

[49] Kono, H., Rusyn, I., Yin, M., Gabele, E. et al., NADPH oxidasederived free radicals are key oxidants in alcohol-induced liver disease. J. Clin. Invest 2000, 106, 867-872.

[50] Ceccarelli, S., Nobili, V., Alisi, A., Toll-like receptor-mediated signaling cascade as a regulator of the inflammation network during alcoholic liver disease. World J. Gastroenterol. 2014, 20, 16443-16451.

[51] Uesugi, T., Froh, M., Arteel, G. E., Bradford, B. U. et al., Tolllike receptor 4 is involved in the mechanism of early alcoholinduced liver injury in mice. Hepatology 2001, 34, 101-108.

[52] Beutler, B., Microbe sensing, positive feedback loops, and the pathogenesis of inflammatory diseases. Immunol. Rev. 2009, 227, 248-263.

[53] Everard, A., Belzer, C., Geurts, L., Ouwerkerk, J. P. et al., Cross-talk between Akkermansia muciniphila and intestinal epithelium controls diet-induced obesity. Proc. Natl. Acad. Sci. U S A 2013, 110, 9066-9071.

[54] Everard, A., Lazarevic, V., Derrien, M., Girard, M. et al., Responses of gut microbiota and glucose and lipid metabolism to prebiotics in genetic obese and diet-induced leptinresistant mice. Diabetes 2011, 60, 2775-2786.

[55] Chang, C. J., Lin, C. S., Lu, C. C., Martel, J. et al., Ganoderma lucidum reduces obesity in mice by modulating the composition of the gut microbiota. Nat. Commun. 2015, 6, 7489.

[56] Delzenne, N. M., Bindels, L. B., Gut microbiota: Ganoderma lucidum, a new prebiotic agent to treat obesity? Nat. Rev. Gastroenterol. Hepatol. 2015, 12, 553-554. 